\title{
Utilidad y complicaciones de la biopsia percutánea esplénica con aguja tru-cut guiada por imágenes*
}

\author{
Drs. CECILIA BESA C. ${ }^{1}$, CRISTÓBAL TEUBER S. ${ }^{1}$, Ints. ÁlVARO HUETE G. ${ }^{2}$, \\ ANDRÉS O'BRIEN S. ${ }^{2}$, FRANCISCO CRUZ O. ${ }^{2}$, ROBERTO OYANEDEL Q. ${ }^{2}$, \\ 1 Departamento de Radiología. Hospital Clínico Pontificia Universidad Católica de Chile. \\ 2 Interno Facultad de Medicina Pontificia Universidad Católica de Chile. \\ Santiago, Chile.
}

\begin{abstract}
Ultrasound guided needle biopsy of the spleen. Review of 13 procedures

Background: Needle biopsies of the spleen were avoided due to the fear of bleeding in a highly vascularized organ. However their safety, even using 18 gauge needles, has been demonstrated. Aim: To report the experience with ultrasound guided needle biopsies of the spleen. Material and Methods: Retrospective review of records of ultrasound guided biopsies of the spleen using Tru-cut ${ }^{\mathrm{TM}}$ needles, performed between 2005 and 2009. Results: Thirteen procedures performed in 12 patients were identified. A specific diagnosis was achieved in nine $(69 \%)$ procedures (lymphoma in four, melanoma in 2, sarcoma in 1, extremedullary erythropoiesis in one and splenic cryptococcosis in one. Two patients with negative results were subjected to a new biopsy, which yielded the diagnosis of lymphoma. A third patient was studied elsewhere, finding a malignant tumor. Two patients had complications, one had a vagal reaction and other had a perisplenic hematoma without clinical repercussion. Conclusions: Ultrasound guided needle biopsy of the spleen is a safe and useful procedure.
\end{abstract}

Key words: Spleen, needle biopsy, lymphoma.

\section{Resumen}

Objetivo: Reportar la experiencia de biopsias percutáneas esplénicas con aguja tru-cut guiadas por imágenes. Materiales y Métodos: Revisión retrospectiva de biopsias esplénicas con aguja tru-cut guiadas por ultrasonido (US) y tomografía computada (TC) realizadas en nuestro hospital desde Enero de 2005 a Abril de 2009. Resultados: Se identificaron un total de 13 procedimientos. La biopsia percutánea logró un diagnóstico específico en $9(69 \%)$ de las 13 intervenciones. Los diagnósticos fueron linfoma $(\mathrm{n}=4)$, melanoma (n $=2)$, sarcoma $(n=1)$, hematopoyesis extramedular $(n=1)$ y criptococosis esplénica $(n=1)$. De las biopsias no diagnósticas 3 casos correspondieron a patología neoplásica y uno a patología benigna. Se reportaron 2 complicaciones (15\%). Discusión: La biopsia esplénica percutánea guiada por imágenes con aguja tru-cut es un procedimiento útil y seguro, capaz de determinar el diagnóstico definitivo en la mayoría de los pacientes y evitar la mayoría de las esplenectomías diagnósticas.

Palabras clave: Bazo, biopsia percutánea, tomografía computada, ultrasonido.

\footnotetext{
*Recibido el 17 de Octubre de 2009 y aceptado para publicación el 14 de Enero de 2010.

Correspondencia: Dra. Cecilia Besa C.

Marcoleta 367, Santiago, Chile. Fono: 3543486 - Fax: 6336762

E-mail: besacecilia@gmail.cl
} 


\section{Introducción}

Las biopsias percutáneas guiadas por imágenes se han convertido en un procedimiento estándar en la aproximación inicial de lesiones en diferentes órganos del cuerpo ${ }^{1}$. Este procedimiento permite en la mayoría de los casos, un diagnóstico definitivo de lesiones tumorales y alteraciones inflamatoriasinfecciosas, evitando procedimientos invasivos ${ }^{2-4}$.

Por bastante tiempo las punciones esplénicas fueron evitadas por tratarse de un órgano con elevado riesgo de hemorragia ${ }^{5}$. Sin embargo, este concepto ha ido cambiando en los últimos 20 años, al demostrarse la seguridad de las punciones con agujas cada vez más gruesas. Numerosos reportes señalan la seguridad de agujas $22,21,20$ y hasta $18-\mathrm{G}^{2,3,5-7}$.

En el caso de la patología esplénica, la biopsia percutánea es un procedimiento efectivo que permite prescindir de la mayoría de las esplenectomías diagnósticas ${ }^{8}$ evitando así la morbimortalidad asociada a esta intervención.

El objetivo de este trabajo es reportar la experiencia de biopsias percutáneas esplénicas con aguja tru-cut guiadas por imágenes (US/TC) en nuestra institución.

\section{Material y Método}

\section{Pacientes}

Se realizó una revisión retrospectiva de las biopsias esplénicas realizadas entre Abril de 2005 y Abril de 2009 en nuestro hospital. Se identificaron un total de 12 pacientes sometidos a un total de 13 biopsias esplénicas percutáneas guiadas por imágenes (US/ TC).

Se obtuvieron los registros médicos, las imágenes radiológicas pertinentes y los resultados de las biopsias. Se recopiló información demográfica, antecedentes clínicos, características de las lesiones en estudio, complicaciones de la punción percutánea y se estableció un diagnóstico definitivo para cada paciente.

Se definieron como complicaciones todos los eventos adversos registrados en el historial de cada paciente previo al alta médica, o durante las 24 horas siguientes al procedimiento en pacientes hospitalizados. Se buscaron dirigidamente hemorragias en control con imágenes post-punción, alteración de signos vitales, presencia de dolor y uso de analgésicos complementarios.

El diagnóstico definitivo de cada paciente fue establecido por el resultado histológico de la biopsia o de la esplenectomía en los casos en que ésta fue realizada. En aquellos con biopsia negativa o inespecífica se realizó un seguimiento clínico para determinar el diagnóstico final.

Se evaluó la utilidad de la intervención mediante

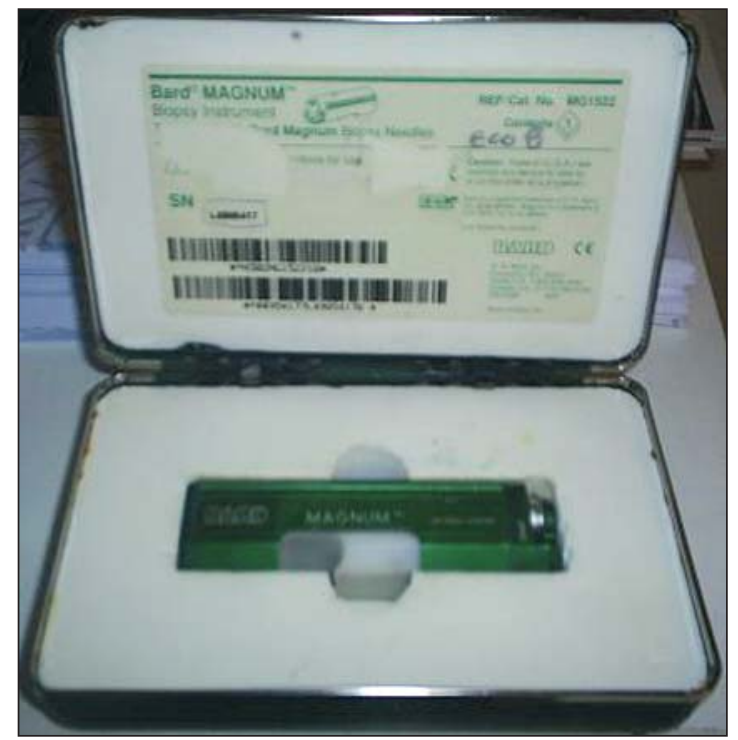

Figura 1. Sistema Coaxial Bard Magnum MG 1522.

la concordancia entre el diagnóstico de la biopsia con el diagnóstico definitivo establecido. Se reportaron las complicaciones identificadas.

\section{Técnica}

Las 13 intervenciones fueron realizadas bajo visión imagenológica: 5 mediante TC y 8 mediante US. Los pacientes fueron hospitalizados el día del procedimiento y se obtuvo un consentimiento informado por escrito de todos los pacientes una vez explicado el procedimiento. En todos los pacientes se realizaron pruebas de coagulación antes de la intervención, con necesidad de plasma fresco congelado en un caso por tiempo de protrombina (TP) de $47 \%$.

Se realizó un control, con la modalidad de imagen correspondiente, previo al procedimiento para identificar las lesiones y la zona de punción. Con técnica aséptica se puncionó utilizando agujas $18-\mathrm{G}$ o 20-G montadas en un sistema coaxial Bard Magnum MG 1522® (Figura 1). Se obtuvieron muestras de 1 a 3 fragmentos de $1-13 \mathrm{~mm}$ de longitud, los cuales fueron enviados en formalina para estudio histológico y en suero fisiológico para citometría de flujo. En las Figuras 2 y 3 se presentan imágenes de 2 procedimientos.

Se realizó un control con imágenes en busca de complicaciones inmediatas. Se mantuvo control de signos vitales durante las primeras 4 horas y se indicó un analgésico cada 6-8 horas y un analgésico adicional a solicitud del paciente en caso de dolor. Los pacientes fueron dados de alta al día siguiente del procedimiento transcurridas $12-24$ horas de observación. 


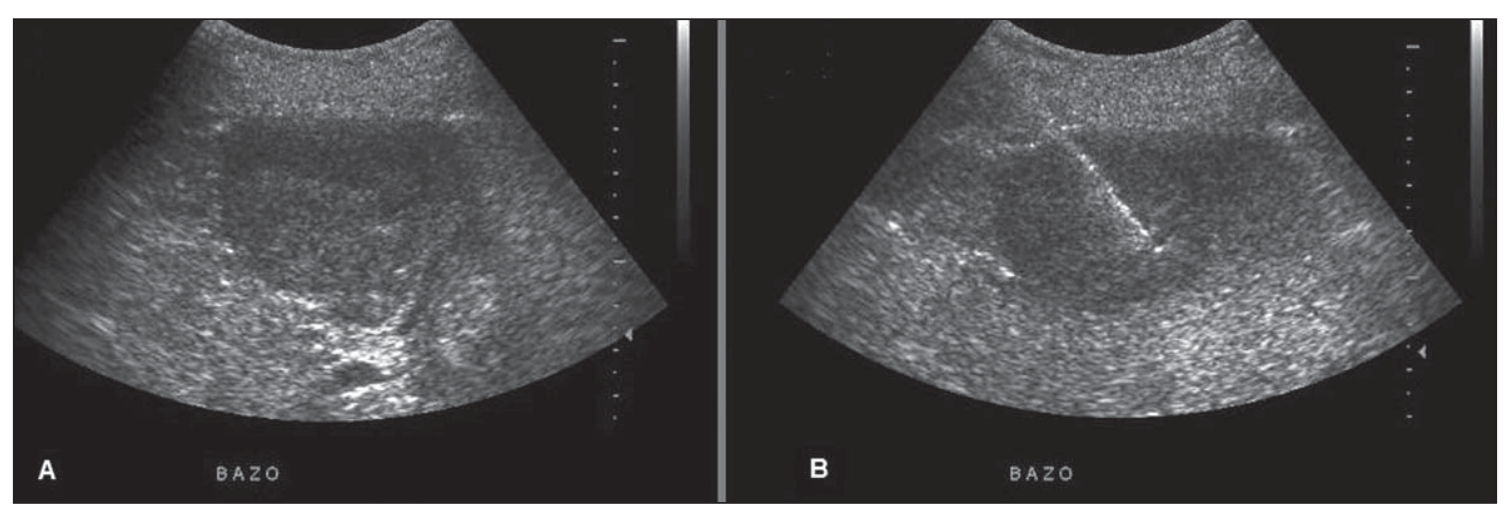

Figura 2. Imágenes axiales de ecografía pre-punción y durante el procedimiento muestran una lesión ovoidea hipoecogénica de bordes bien definidos cuyo diagnóstico histológico demostró una metástasis de melanoma.
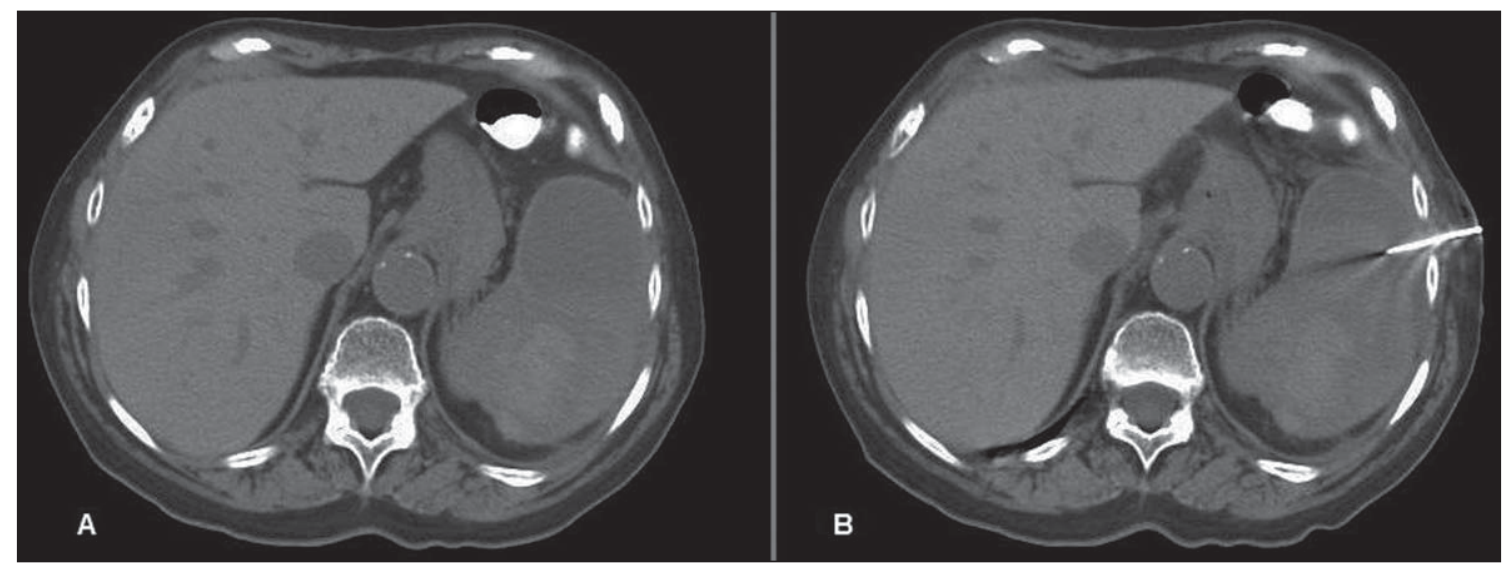

Figura 3. Imágenes axiales de TC pre-punción y durante el procedimiento muestran una lesión focal esplénica hipodensa que correspondió a un linfoma.

\section{Resultados}

De los 12 pacientes, 10 fueron hombres y 2 mujeres; sus edades fluctuaron entre 31 y 80 años con un promedio de 58 años. Dos pacientes tenían antecedentes de patología neoplásica: un linfoma y un melanoma. Un paciente fue inmunosuprimido por VIH y un paciente tenía antecedente de mielofibrosis. Los 8 pacientes restantes no tuvieron un diagnóstico establecido al momento de la intervención.

Se obtuvo biopsia de 12 lesiones focales cuyo tamaño fluctuó entre 1,8 y $8,5 \mathrm{~cm}$ con un promedio de $3,7 \mathrm{~cm}$. En un caso, la muestra histológica, fue obtenida de una esplenomegalia sin lesiones focales. La Tabla 1 presenta las características y el tamaño de las lesiones puncionadas.

La biopsia percutánea logró un diagnóstico específico en $9(69,2 \%)$ de las 13 intervenciones.
En $7(78 \%)$ casos se diagnosticó una patología maligna, incluyendo 4 linfomas, 2 melanomas y 1 sarcoma. El resultado histológico fue benigno en 2 casos (22\%), incluyendo 1 hematopoyesis extramedular y 1 criptococosis esplénica. En la Tabla 2, se presentan los antecedentes de cada paciente, la técnica utilizada para la punción y los diagnósticos establecidos.

En 4 casos la biopsia percutánea no obtuvo el diagnóstico final establecido. Dos de estos casos (casos 3 y 7), fueron sometidos posteriormente a una segunda biopsia esplénica percutánea obteniendo un diagnóstico definitivo; los dos casos correspondieron a linfoma. De los dos casos restantes, uno (caso 1) fue estudiado en un centro externo diagnosticándose una neoplasia esplénica maligna, a cuyo informe histológico no tuvimos acceso, y en el otro (caso 11) no se logró establecer un diagnóstico definitivo ya que 
UTILIDAD Y COMPLICACIONES DE LA BIOPSIA PERCUTÁNEA ESPLÉNICA CON AGUJA TRU-CUT GUIADA POR...

Tabla 1. Características de las lesiones puncionadas

\begin{tabular}{ccccc}
\hline Caso & Esplenomegalia & n lesiones* $^{*}$ & Adenopatías** $^{* *}$ & Lesión puncionada $(\mathbf{m m})$ \\
\hline 1 & + & 1 & - & 85 \\
2 & - & 3 & - & 7 \\
3 & + & 5 & + & 18 \\
4 & + & 0 & + & 0 \\
5 & + & 5 & - & 30 \\
6 & + & 5 & - & 27 \\
7 & + & 5 & + & 12 \\
8 & + & 5 & + & 12 \\
9 & - & 1 & - & 25 \\
10 & + & 5 & + & 47 \\
11 & + & 5 & + & 30 \\
12 & + & 5 & - & 27 \\
13 & + & 1 & - & 55 \\
\hline
\end{tabular}

* Cinco equivale a 5 o más. ** Adenopatías retroperitoneales.

Tabla 2. Antecedentes, técnica utilizada y diagnósticos establecidos en cada caso

\begin{tabular}{cclccll}
\hline Caso & $\begin{array}{c}\text { Edad/ } \\
\text { Sexo }\end{array}$ & Antecedentes & Técnica & $\begin{array}{c}\text { Aguja } \\
(\mathbf{G})\end{array}$ & Diagnóstico biopsia & Diagnóstico definitivo \\
\hline 1 & $80 / \mathrm{F}$ & No & TC & 18 & Fibrosis intersticial & Neoplasia maligna esplénica* \\
2 & $31 / \mathrm{M}$ & VIH & TC & 18 & Criptococosis & Criptococoma esplénico \\
3 & $59 / \mathrm{M}$ & No & US & 18 & Artefacto desecación y fijación & Linfoma de Hodgkin \\
4 & $42 / \mathrm{M}$ & No & US & 18 & Linfoma de células T & Linfoma de células T \\
5 & $60 / \mathrm{M}$ & No & US & 18 & Linfoma de Hodgkin & Linfoma de Hodgkin \\
6 & $72 / \mathrm{M}$ & No & US & 18 & Linfoma de células B & Linfoma de células B \\
7 & $64 / \mathrm{M}$ & Linfoma & TC & 18 & Pulpa roja & Linfoma de células B \\
8 & $65 / \mathrm{M}$ & Linfoma & US & 18 & Linfoma de células B & Linfoma de células B \\
9 & $44 / \mathrm{M}$ & Melanoma & US & 18 & Melanoma metastásico & Melanoma \\
10 & $56 / \mathrm{M}$ & No & US & 18 & Melanoma metastásico & Melanoma \\
11 & $57 / \mathrm{F}$ & No & US & 18 & Inflamación granulomatosa* & Síndrome mielodisplásico \\
12 & $59 / \mathrm{M}$ & No & US & 18 & Sarcoma de alto grado & Sarcoma de alto grado \\
13 & $69 / \mathrm{M}$ & Mielofibrosis & TC & 20 & Hematopoyesis extramedular & Hematopoyesis extramedular \\
\hline
\end{tabular}

* No especificada.

el paciente falleció por otra causa antes de someterse a una esplenectomía diagnóstica.

Se registraron $2(15,3 \%)$ complicaciones: una reacción vagal postpunción que requirió una dosis de atropina y un pequeño hematoma periesplénico sin repercusión clínica. Ningún paciente solicitó analgesia adicional.

\section{Discusión}

La efectividad de las biopsias esplénicas percutáneas reportada en nuestro estudio $(69,2 \%)$, se acerca a la efectividad diagnóstica reportada en otras series que fluctúan entre 75 y $88,9 \% \%^{3,4,6}$. El menor rendimiento reportado en nuestra serie puede verse 
explicado por la pérdida de una de las muestras por desecación y el limitado tamaño muestral.

Dos de los pacientes con resultados inespecíficos en la biopsia fueron sometidos a una segunda biopsia esplénica percutánea, con lo cual se logró un diagnóstico definitivo, aumentando así la efectividad de la técnica. Esto concuerda con lo reportado por otros autores, en que una segunda biopsia aumenta la probabilidad de obtener el diagnóstico definitivo, especialmente en pacientes con sospecha de linfoma ${ }^{3}$.

No se realizó ninguna esplenectomía diagnóstica en nuestra serie, sin embargo, ésta estaba indicada en un caso, la que no se realizó por el fallecimiento del paciente. Dos pacientes tuvieron indicación de esplenectomía terapéutica.

El porcentaje de complicaciones reportado $(15,3 \%)$ es similar al de otras series recientes s.5, $^{3,5,10}$ destacando que no se observaron complicaciones clínicamente relevantes. Las complicaciones graves, principalmente hemorrágicas, se han reportado en menos del 1\% de las intervenciones ${ }^{9}$. A la fecha no hemos encontrado referencias nacionales al respecto.

La principal limitación de nuestro estudio fue el pequeño tamaño muestral lo que probablemente influyó en los resultados, considerando que una de las muestras presentó artefacto de desecación y fijación disminuyendo la efectividad diagnóstica.

En conclusión, la biopsia esplénica percutánea guiada por imágenes con aguja tru-cut, en concordancia con otras series recientes, es un procedimiento útil y seguro, capaz de determinar el diagnóstico definitivo en la mayoría de los pacientes y evitar la mayoría de las esplenectomías diagnósticas.

\section{Agradecimientos}

Al Departamento de Anatomía Patológica de la Facultad de Medicina de la Pontificia Universidad Católica de Chile.

\section{Referencias}

1. Contreras O, Burdiles A. Diagnóstico de lesiones óseas con biopsia percutánea guiada por imágenes. Rev Med Chile 2006; 134: 1283-1287.

2. Muraca S, Chait P, Connolly B, Baskin K, Temple M. Us-guided core biopsy of the spleen in children. Radiology 2001; 218: 200-206.

3. Lieberman S, Libson E, Maly B, Lebensart P, BenYehuda D, Bloom AI. Imaging-guided per cutaneous splenic biopsy using a 20- or 22 gauge cutting-edge core biopsy needle for the diagnosis of malignant lymphoma. AJR 2003; 181: 1025-1027.

4. López J, Del Cura J, Fernández de Larrinoa A, Gorriño O, Zabala R, Bilbao F. Role of ultrasound-guided core biopsy in the evaluation of spleen pathology. APMIS 2006; 114: 492-499.

5. Keogan M, Freed K, Paulson E, Nelson R, Dodd L. Imaging-guided percutaneous biopsy of focal splenic lesions: update on safety and effectiveness. AJR 1999; 172: 933-937.

6. Liang P, Gao Y, Wang Y, Yu X, Yu D, Dong B. USguided percutaneous needle biopsy of the spleen using 18-gauge versus 21-gauge needles. J Clin Ultrasound 2007; 35: 477-482.

7. Kang M, Kalra N, Gulati M, Lal A, Kochhar R, Rajwanshi A. Image guided percutaneous splenic interventions. Eur J Radiol 2007; 64: 140-146.

8. Lindgren P, Hagberg H, Eriksson B, Glimelius B, Magnusson A, Sundstrom C. Excision biopsy of the spleen by ultrasound guidance. Br J Radiol 1985; 58: 853-857.

9. Civardi G, Vallisa D, Berte R, Giorgio A, Filice C, Caremani $\mathrm{M}$, et al. Ultrasound-guided fine needle biopsy of the spleen: high clinical efficacy and low risk in a multicenter italian study. Am J Hematol 2001; 67: 93 99.

10. Lal A, Ariga R, Gattuso P, Nemcek A, Nayar R. Splenic fine needle aspiration and core biopsy. A review of 49 cases. Acta Cytol 2003; 47: 951-959. 